\title{
Desarrollo de un Protocolo de Plastinación de Cortes con Resina Poliéster Aplicado a Secciones de Cerebro Humano
}

\author{
Development of a Sheet Plastination Protocol with Polyester Resin Applied to Human Brain Slices
}

Marco Guerrero $^{1,2}$; Claudia Vargas ${ }^{1,3}$; Eduardo Alarcón ${ }^{4}$ Mariano del Sol $^{1,5}$ \& Nicolás Ernesto Ottone ${ }^{1,5,6}$

GUERRERO, M.; VARGAS, C.; ALARCóN, E.; DEL SOL, M. \& OTTONE, N. E. Desarrollo de un protocolo de plastinación de cortes con resina poliéster aplicado a secciones de cerebro humano. Int. J. Morphol., 37(4):1557-1563, 2019.

RESUMEN: La plastinación es una técnica anatómica de conservación cadavérica creada en 1977 por Gunther von Hagens, en Heidelberg, Alemania, y que sustituye los líquidos biológicos y/o de fijación por acetona, para luego impregar las muestras con distintas resinas, dependiendo de la técnica de plastinación desarrollada, para finalmente llevar a cabo la polimerización de los componentes incorporados a las muestras, para obtener muestras biológicas secas y totalmente duraderas. El objetivo de este trabajo consistió en desarrollar un protocolo de plastinación de cortes con resina poliéster (Biodur® P40) en secciones de 3 mm de espesor de cerebro humano. La muestras fueron fijadas y conservadas con formalina al $10 \%$. Los cerebros luego fueron seccionados con una maquina cortadora de tejidos, obteniéndose láminas delgadas de $3 \mathrm{~mm}$ de espesor. Inmediatamente los cortes de cerebro fueron colocados en deshidratación en acetona al $100 \%$, a $-25^{\circ} \mathrm{C}$, durante 7 días el primer baño de acetona, y durante otros 3 días más, para el segundo baño de acetona. Una vez deshidratados los cortes, estos fueron colocados en resina poliéster Biodur® P40 y se llevó a cabo la impregnación forzada de los cortes, en cámara de vacío a temperatura ambiente $\left(20^{\circ} \mathrm{C}\right)$. Una vez finalizada la impregnación forzada, se procedió a la etapa de curado, la cual en primer lugar consiste en el armado de las cámaras de curado dentro de las cuales se colocaran los cortes con resina poliéster. Las cámaras de curado fueron colocadas bajo luz UV para acelerar la polimerización del poliéster y finalizar el proceso de plastinación. Se logró desarrollar satisfactoriamente en el Laboratorio de Plastinación y Técnicas Anatómicas de la Universidad de La Frontera un protocolo de plastinación de cortes con resina poliéster, obteniendo una excelente conservación de cortes de cerebro, con diferenciación de sustancias gris y blanca, y conservación de todas las características morfológicas.

PALABRAS CLAVE: Plastinación de Cortes; Resina poliéster.

\section{INTRODUCCIÓN}

El desarrollo de técnicas anatómicas para fijar y preservar vivió una revolución por la aparición de la técnica de plastinación, creada por el profesor Gunther von Hagens en 1977 en Heidelberg, Alemania, y posteriormente diversificada y desarrollada por varios centros de investigación en los últimos años (Fasel et al., 1988; Cook \& Al-Ali, 1997; Steinke, 2001; Shahar et al., 2007; Sora et al., 2003; Sora \& Cook, 2007; Al-Ali et al., 2009 ; Soal et al., 2010; Ottone, 2013; Scali et al., 2015; Ottone et al., 2014, 2015, 2016, 2018a,b; Ottone, 2018; Prieto et al., 2019). La técnica de plastinación consiste en la eliminación de los líquidos de fijación de las muestras biológicas, por lo general formalina, y su reempla- zo en una primera etapa por acetona, con su correspodiente proceso de deshidratación. Posteriormente, la acetona es sustituida por medio de un proceso de impregnación forzada al vacío (ya sea en frío o temperatura ambiente) por una mezcla de silicona con su correspondiente catalizador. Finalmente, una vez reemplazada toda la acetona por esta mezcla de silicona-catalizador, la muestra es sometida a un proceso de curado, en el cual se acelera la polimerización de la mezcla, para lograr alcanzar el objetivo final de la técnica, que es es obtener preparaciones secas con cierto grado de rigidez y/o flexibilidad y que pueden mantenerse durante largos períodos de tiempo (Ottone et al., 2015, 2018b).

\footnotetext{
${ }^{1}$ Programa de Doctorado en Ciencias Morfológicas, Facultad de Medicina, Universidad de La Frontera, Temuco, Chile.

${ }^{2}$ Cátedra de Anatomía Normal, Facultad de Ciencias Médicas, Universidad Central del Ecuador, Quito, Ecuador.

${ }^{3}$ Departamento de Educación Física Deportes y Recreación, Facultad de Educación y Humanidades, Universidad de La Frontera, Temuco, Chile.

${ }^{4}$ Unidad de Anatomía Normal, Universidad de Santiago de Chile, Santiago, Chile.

${ }^{5}$ Laboratorio de Plastinación y Técnicas Anatómicas, Centro de Investigación en Ciencias Odontológicas (CICO), Facultad de Odontología, Universidad de La Frontera, Temuco, Chile.

${ }^{6}$ Centro de Excelencia en Estudios Morfológicos y Quirúrgicos (CEMyQ), Facultad de Medicina, Universidad de La Frontera, Temuco, Chile.
} 
La plastinación de cortes con resina poliéster fue diseñada por von Hagens para conservar permanentemente cortes de tejido, inicialmente cerebral, dentro de una lámina rígida y duradera de resina curada (von Hagens et al., 1987). Posteriormente, esta técnica también comenzó a usarse para plastinar otras regiones corporales (Ottone, 2018; Ottone et al., 2018b).

El objetivo del presente trabajo es desarrollar el protocolo clásico de plastinación de cortes con resina poliéster (Biodur ${ }^{\circledR}$ P40) de secciones de cerebro, estandarizando su uso en muestras humanas, estableciendo detalles característicos y de diferencianción en los protocolos desarrollados por otros grupos de investigación.

\section{MATERIAL Y MÉTODO}

El presente trabajo se realizó en el Laboratorio de Plastinación y Técnicas Anatómicas, de la Facultad de Odontología de la Universidad de La Frontera (UFRO), Temuco, Chile. A su vez, se ha contado con el apoyo del Centro de Excelencia en Estudios Morfológicos y Quirúrgicos (CEMyQ) y del Programa de Doctorado en Ciencias Morfológicas.

El protocolo de plastinación de cortes con resina poliéster fue realizado con equipamiento desarrollado por el Laboratorio de Plastinación y Técnicas Anatómicas (UFRO). El poliéster utilizado fue adquirido a la empresa Biodur (Biodur ${ }^{\circledR} \mathrm{P} 40$ ). El resto de los insumos fueron obtenidos localmente en Chile.

\section{Protocolo de Plastinación de Cortes con Resina Poliéster}

Fijación de las muestras. Los cerebros, para asegurar una consistencia adecuada, y evitar una excesiva retracción durante el proceso de plastinación, requieren ser fijados en formalina al $20 \%$, durante un mínimo de 60 días. En este sentido, lo ideal es comenzar con concentraciones de 5, 10, $15 \%$ de formalina, con cambios sucesivos iniciales de una semana, alcanzando la cuarta semana una concentración de formalina al $20 \%$, dejando la muestra en esta concentración durante 30 días más. Se debe eliminar de los cerebros, previo a la fijación, la duramadre y todos los vasos sanguíneos, para facilitar la penetración de la formalina en el tejido nervioso. La fijación debe realizarse por inmersión.

Lavado de las muestras. Previo al corte, los cerebros deben ser lavados en agua corrientes durante un mínimo de una semana, para eliminar el exceso de formalina presente en los tejidos previo a la sección de los mismos.
Sección de muestras. Los cerebros fueron seccionados en láminas de 3 de espesor con una maquina cortadora con sierra de acero inoxidable. Los cortes se colocaron entre rejillas perforadas cuadriculadas, superponiédolas una encima de la otra y quedando entre medio las secciones de cerebro y cerebelo, manteniéndo los cortes separados, para que puedan deshidratarse correctamente. Estas rejillas deben ser anudadas con hilo de pescar, formando un paquete, para asegurar que no se desplacen los cortes (Fig. 1). Este paquete que queda armado es sumergido posteriormente en la acetona, para dar comienzo al proceso de deshidratación, y sin desarmarlo, una vez finalizada la deshidratación, este paquete se sumergirá en la resina poliéster para el proceso de impregnación forzada.

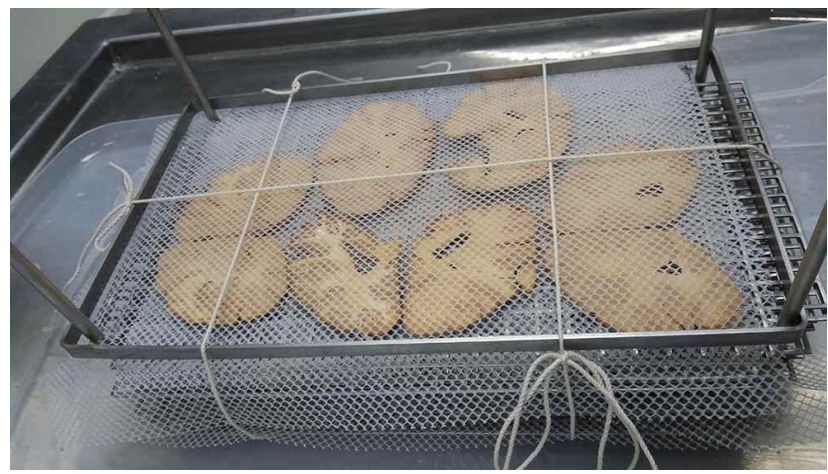

Fig. 1. Muestras dispuestas entre rejillas perforadas conformando, en su conjunto, un paquete que permite movilizar todas las muestras en conjunto evitando el desplazamiento de los cortes, y asegurando su correcta deshidratación y posteriormente impregnación.

Deshidratación. Se deshidrataron las secciones de cerebro en dos baños consecutivos de acetona al $100 \%$, a $-25^{\circ} \mathrm{C}$. El porcentaje de acetona debe controlarse durante todo el proceso de deshidratación, que dura 10 días. La duración del primer baño de acetona fue de 7 días, reduciéndose el porcentaje de acetona en $97 \%$. El segundo baño de acetona duró 3 días, alcanzando la acetona un porcentaje final de $99,5 \%$, con lo cual se dio por finalizada esta etapa.

Impregnación forzada. Este paso consiste en la extracción de la acetona y su reemplazo por resina poliéster mediante la generación de vacío dentro de una cámara de vacío a temperatura ambiente $\left(20-22^{\circ} \mathrm{C}\right)$. Se lleva a cabo la reducción de la presión, durante 24 horas, desde $760 \mathrm{mmHg}$ hasta los $20 \mathrm{mmHg}$. Aplicamos el proceso de impregnación forzada activa-pasiva (Ottone et al., 2015), con dos fases de 8 horas de impregnación forzada activa (16 horas en total con bomba de vacío activada) separadas por una fase intermedia de 8 horas de impregnación forzada pasiva (con bomba de vacío apagada). La extracción de la acetona y su reemplazo por la resina poliéster es visualizada por la aparición de burbujas, que es la acetona que está siendo extraída (Fig. 2). 


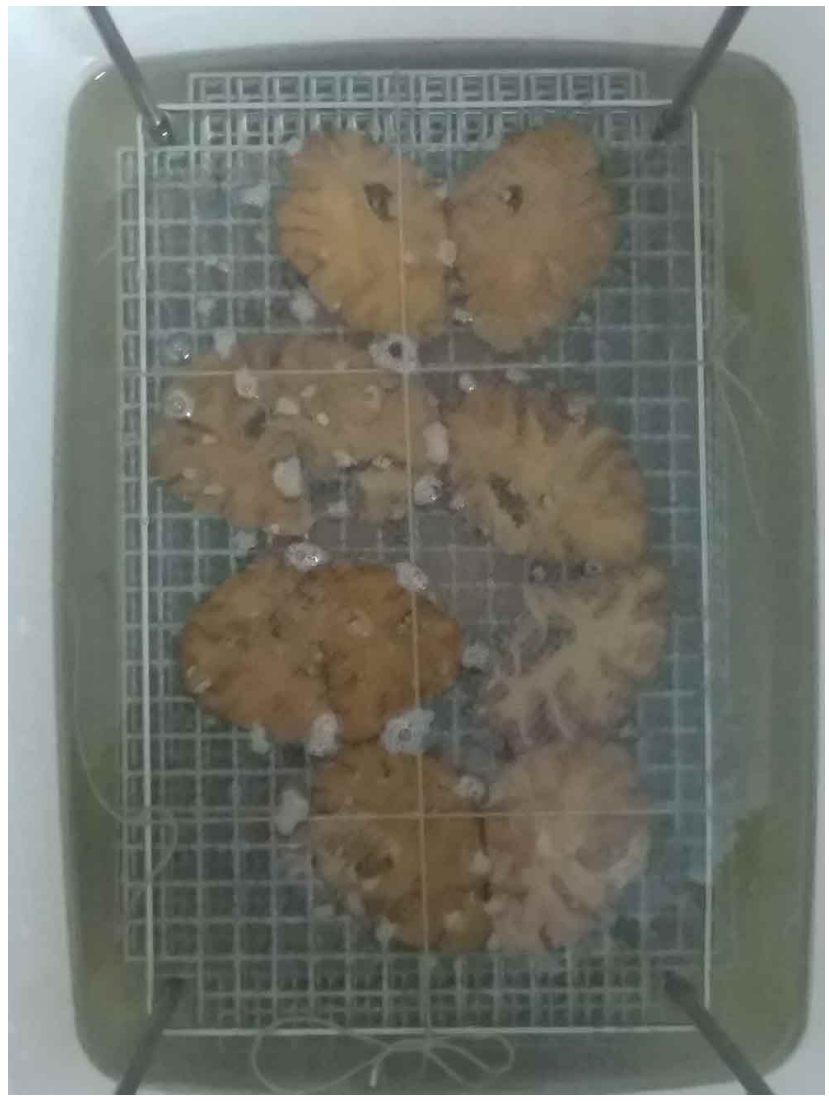

Fig. 2. Burbujeo característico de la impregnación forzada, en la cual las burbujas representan la acetona que está siendo extraída del interior de los cortes, ocupando su espacio la resina poliéster para asegurar la impregnación de las muestras.

Una vez finalizado el burbujeo ha finalizado la impregnación forzada, ocurriendo esto al alcanzar los $20 \mathrm{mmHg}$, en la parte final de la segunda fase de 8 horas de impregnación forzada activa (Tabla I).

Tabla I. Proceso de impregnación forzada activa-pasiva.

\begin{tabular}{lcc}
\hline $\begin{array}{l}\text { Fase Impregnación } \\
\text { Forzada }\end{array}$ & $\begin{array}{c}\text { Tiempo } \\
\text { (horas) }\end{array}$ & $\begin{array}{c}\text { Presión } \\
(\mathrm{mmHg})\end{array}$ \\
\hline Activa & 8 & $760-150$ \\
Pasiva & 8 & 150 \\
Activa & 8 & $150-20$ \\
\hline
\end{tabular}

Armado de cámaras planas de curado. Una vez finalizada la impregnación forzada, los cortes están listos para ser incorporados en las cámaras planas de curado (Fig. 3). Los cortes de cerebro y cerebelo fueron extraídos de la resina poliéster y fueron colocados dentro de cámaras planas para llevarlos a la etapa de curado. Estas cámaras planas consistieron en dos placas de vidrio de $3 \mathrm{~mm}$ de espesor, las cuales se enfrentaron entre sí separadas por una goma de silicona

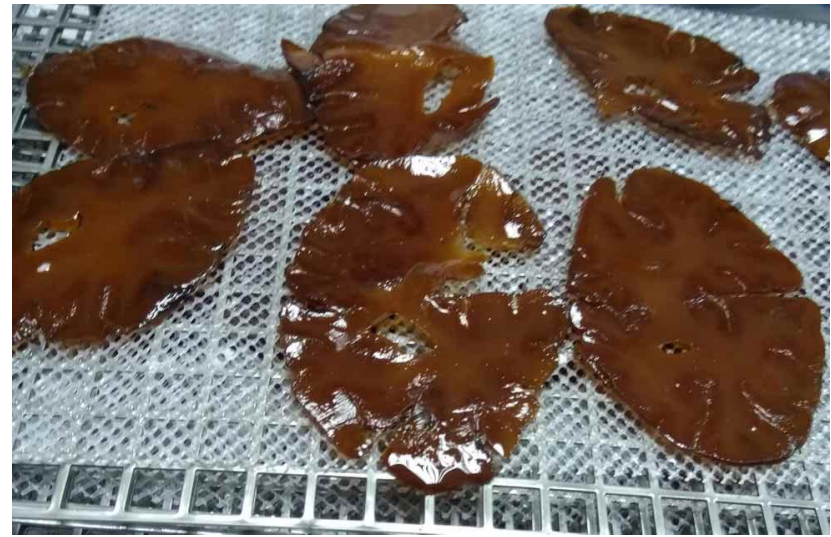

Fig. 3. Cortes de cerebro luego de finalizado el proceso de impregnación forzada, visualizándose la correcta impregnación de los mismos.

de $5 \mathrm{~mm}$ de espesor, y se mantuvieron juntas utilizando broches de papel, los cuales fueron colocados en todos los lados de los vidrios, abarcando vidrio + goma de silicona + vidrio (Fig. 4). De esta manera quedó armada la cámara con una abertura superior, a través de la cual se incorporó el poliéster y luego los cortes de cerebro y cerebelo correspondientes. Antes de cerrar la cámara de curado, se introdujo una bolita de acero, la cual tiene como función, una vez cerrada la cámara de curado, y al aplicar sobre la misma un imán, la movilización de los cortes de muestra para permitir su correcto posicionamiento dentro de la cámara de curado (Fig. 5).

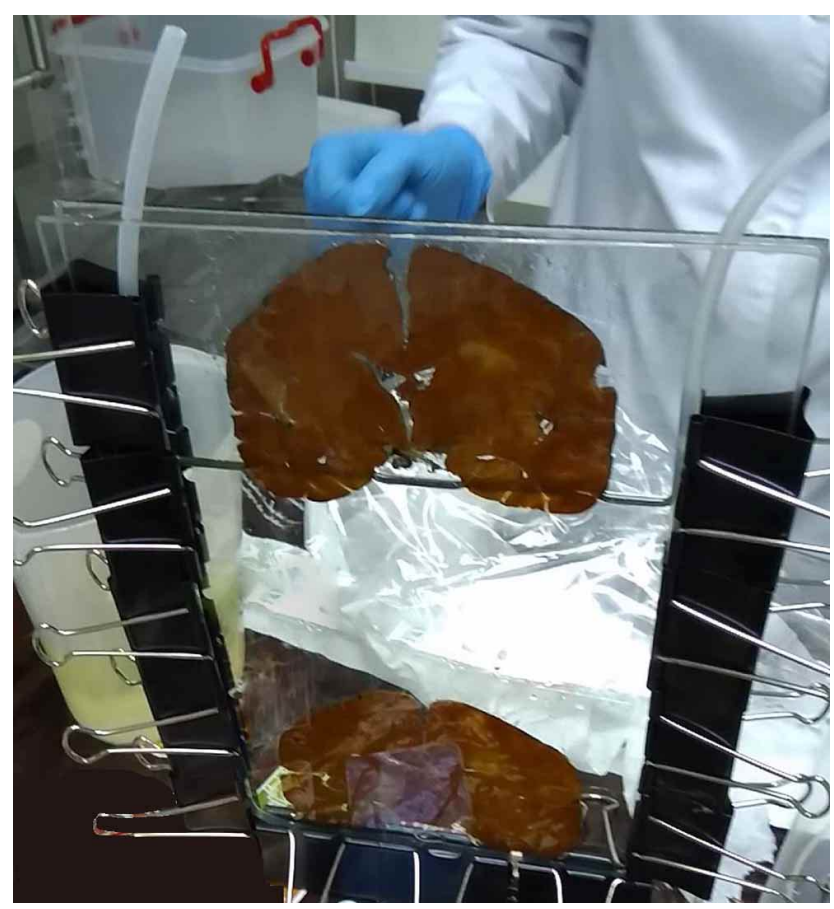

Fig. 4. Incorporación de los cortes de cerebro dentro de las cámaras planas de curado. Observar el llenado con poliéster dentro de las mismas cámaras. 


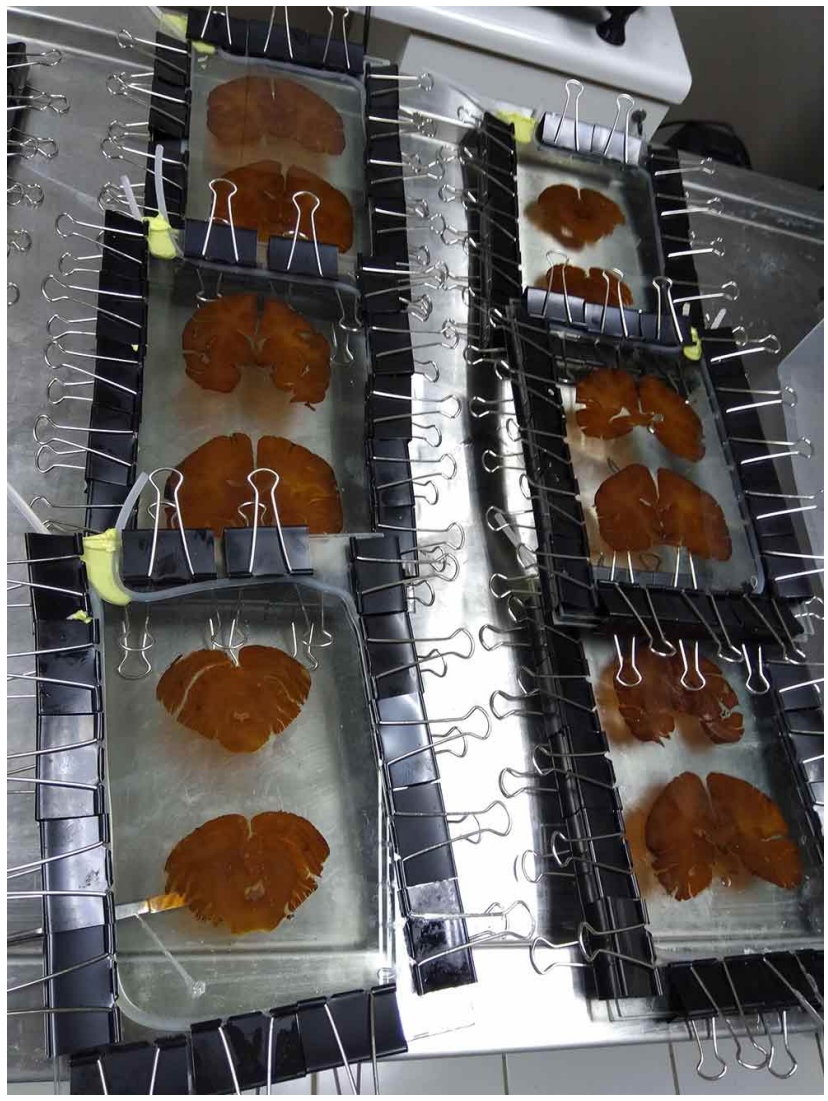

Fig. 5. Cámaras de curado cerradas, con el material anatómico en su interior, listas para ser colocadas en exposición directa a luz ultravioleta para el proceso de curado.
Curado. Las cámaras planas de curado fueron colocadas en luz UV para acelerar el proceso de polimerización de la resina poliester y obtener un endurecimiento de la misma (Fig. 6). En 12 horas se logró endurecer la resina poliester con las muestras en su interior (Fig. 7).

\section{RESULTADOS}

Se logró desarrollar correctamente el protocolo de plastinación de cortes con resina poliéster, estableciendo diferencias en el proceso de impregnación forzada. A partir de este protocolo se obtuvieron cortes de cerebro conservados en su morfología, con una clara diferenciación de la sustancia gris y blanca, con conservación de las características morfológicas de las muestras. Los cortes conservados en resina curada permiten su fácil y biosegura manipulación, sin necesidad de utilizar protección personal para su uso.

\section{DISCUSIÓN}

Los protocolos de plastinación de cortes con resina poliéster, si bien fueron destinados en su inicio para la preservación de cortes de cerebro, por su finalidad de diferenciación de sustancia gris y sustancia blanca, también puede ser extendida a cualquier región anatómica, teniendo en cuen-
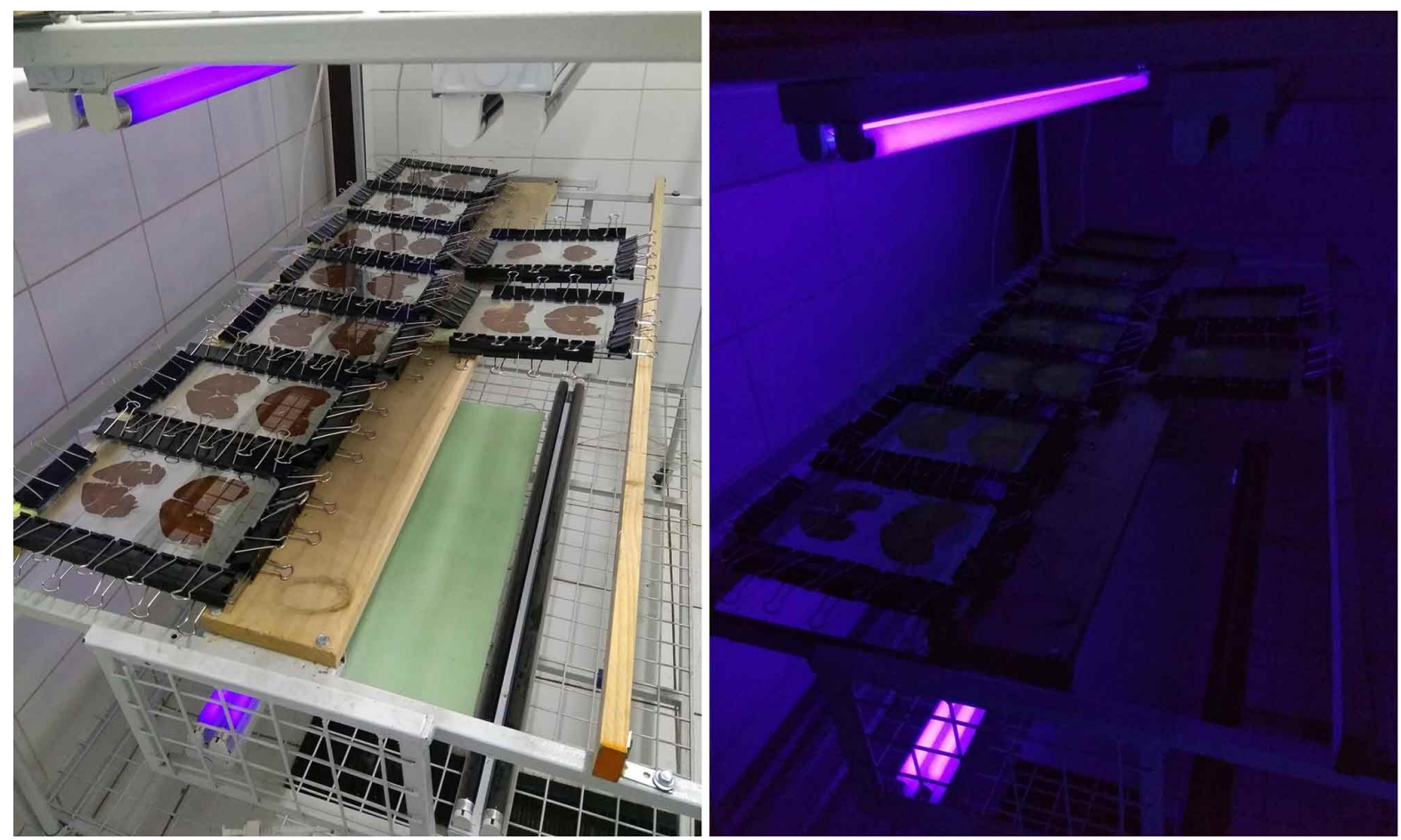

Fig. 6. Cámaras de curado colocadas en el sistema de exposición a luz ultravioleta. 


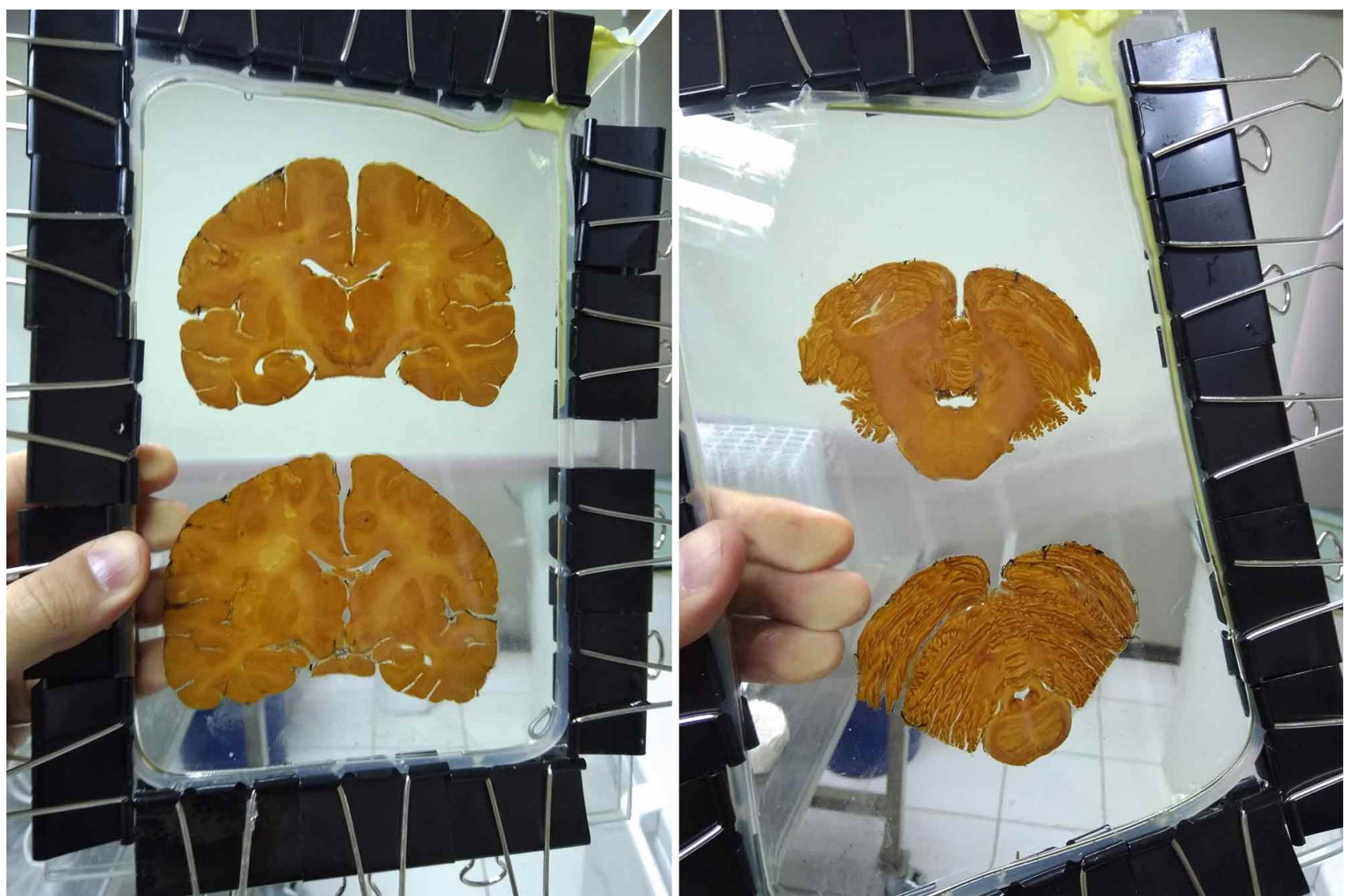

Fig. 7. Proceso de plastinación de cortes con resina poliéster finalizado, visualización de los cortes de cerebro y cerebelo listos para ser utilizados en docencia e investigación.

ta que en comparación a la plastinación de cortes con resina epoxi, el poliéster determina una mayor retracción de los tejidos, sin una mayor transparentación de los tejidos (Ottone et al., 2018). Sin embargo se logran excelentes resultados.

Con respecto al paso de la deshidratación, ésta debe realizarse a baja temperatura $\left(-25\right.$ a $\left.-20{ }^{\circ} \mathrm{C}\right)$ con concentraciones de acetona superiores al $90 \%$, idealmente $100 \%$, en todos los baños de acetona, debiéndose alcanzar finalmente una concentración superior al $99 \%$ para finalizar el proceso de deshidratación. El objetivo de realizar la deshidratación a bajas temperaturas es para reducir la retracción del tejido cerebral, lo cual igual ocurre pero en menor medida, por efecto de la acetona, la que a temperatura ambiente también posee una gran capacidad de eliminación de grasa, lo cual contribuiría a una gran retracción del tejido cerebral si este se deja a temperatura ambiente. Sora \& Brugger (2000) realizaron un interesante trabajo en el cual reemplazan el uso de acetona por metanol. Estos autores relacionan la dificultad en el uso de acetona por los laboratorios debido a condiciones de bioseguridad, especialmente los riesgos de explosición (Sora \& Brugger). Sin embargo, en nuestra re- gión latinoamericana, hay diversos países en los cuales se dificulta el acceso a la acetona por medidas gubernamentales, prohibiendo su uso para investigación científica. De acuerdo a las características de los componentes utilizados en plastinación, es necesario que el disolvente intermediario ideal para la deshidratación tenga una alta presión de vapor y debe ser soluble en agua, características que cumple la acetona, y por lo cual es la seleccionada en esta técnica. Sin embargo, Sora \& Brugger, investigando en una alternativa a la acetona, encontraron en el metanol un componente soluble en agua y con alta presión de vapor, similar a la acetona. En este sentido, el metanol posee un mayor punto de ebullición y menor presión de vapor, características que lo hacen menos volátil que la acetona (Sora \& Brugger). Además, el punto de inflamación de la acetona es significativamente más bajo que el punto de inflamación del metanol. Un hecho que hace que la acetona sea más peligrosa que el metanol. Sin embargo, su extracción durante la etapa de impregnación forzada es algo más compleja, requiriendo un manejo y regulación precisa de las presiones, algo que estos autores solo recomiendan para plastinadores experimentados (Sora \& Brugger). 
Con respecto a la impregnación forzada, en la técnica P40 esta suele realizarse a temperatura ambiente $\left(20^{\circ} \mathrm{C}\right)$, debiendo alcanzar una presión de al menos $20 \mathrm{mmHg}$ para la finalización de la impregnación, en 24 horas. Puede lograrse en menos tiempo la impregnación activa, como en nuestro caso, con un período de impregnación pasiva y con apagado de la bomba de vacío. Durante todo el proceso debe mantenerse cubierta la cámara de vacío, sin que la luz ultravioleta actúe sobre el poliéster, para evitar el comienzo de polimerización de la resina, y el oscurecimiento de los cortes cerebrales. Realizamos la impregnación sólo con la resina poliéster, sin el uso de catalizador. Esto permite la reutilización de la resina, lo que permite reducir costos en la preparación de muestras en gran cantidad.

Por otro lado, la impregnación forzada se realizó en un total de 16 horas de impregnacion forzada activa ( 8 horas de actividad en dos días consecutivos) con un período de 12 horas de impregnación forzada pasiva (apagado de bomba de vacío), diferenciándose de las técnicas clásicas de plastinación de cortes con resina poliéster, en las cuales la impregnación forzada en continua, sin apagado de la bomba de vacío (von Hagens et al., 1987).

En relación al curado, existen protocolos en los cuales combinan el poliéster P40 con un catalizador. Nosotros, de acuerdo a nuestra experiencia, no consideramos necesario combinar el poliéster con un catalizador. Sin embargo, en aquellos protocolos que si los combinan, es fundamental asegurar una correcta fijación de los cerebros. Ya que una deficiente fijación, determinará la aparición de manchas anaranjadas en la corteza cerebral, las cuales se deben a la presencia de peroxidasa activa en el tejido cerebral, que reacciona con el catalizador en el polímero P40. Por lo tanto, esta peroxidasa puede ser inactivada en gran medida por los fijadores, y por esta razón, una fijación completa (formalina $10 \%)$ es vital para el éxito con la técnica P40 en combinación de un catalizador (Barnett, 1997).

Gao et al. (2006) han publicado un protocolo de plastinación con la resina poliéster P45 de la empresa china Hoffen. Esta empresa, cuyo presidente es el Prof. Dr. Sui, presenta una alternativa de producto a Biodur. En esta publicación, han presentado la plastinación de cortes de un delfín, presentando una novedad en su protocolo, en el paso de curado, donde reemplazaron el uso de luz UV por un baño en agua. Con este nuevo nuevo método de curado lograron reducir el espacio requerido para curar las muestras al posicionarlas verticalmente, además de que el baño de agua aseguró la misma dispersión de temperatura en todas las muestras durante el curado, evitando que las temperaturas aumenten demasiado, siendo las temperaturas uniformes en todos los cortes (Gao et al., 2006).
Por su parte, Reed et al. (2008) compararon en su trabajo distintos métodos para acelerar la polimerización y lograr el curado de las muestras. En esta investigación, concluyeron que las fuentes de luz ultravioleta proporcionaron las tasas de curado más rápidas, en comparación a la luz solar y otras fuentes de luz ultravioleta artificial. Por ejemplo, encontraron que la exposición de las muestras a la sombra de la luz solar duplicó el tiempo de curado, en comparación a la exposición directa a otras fuentes de luz UV. Esto mismo sucede al exponer directamente a lámparas de vapor de mercurio. En todos los casos, es fundamental controlar la temperatura, ya que la exposición directa las fuentes de luz $\mathrm{UV}$, sin control de la temperatura, provocaría la ruptura del vidrio de las cámaras de curado (Reed et al., 2008). Es importante que no se superen los $30^{\circ} \mathrm{C}$ (Ottone, 2018), por encima de esta temperatura, hay riesgo de ruptura del vidrio. Reed et al. (2008) también descubrieron que la iluminación fluorescente no tenía ningún efecto sobre el curado de la resina $\mathrm{P} 40$, sin observación de aumento de temperatura, ni reacción exotérmica, como ocurre en el curado de la resina P40.

GUERRERO, M.; VARGAS, C.; ALARCÓN, E.; DEL SOL, M. \& OTTONE, N. E. Development of a sheet plastination protocol with polyester resin applied to human brain slices. Int. J. Morphol., 37(4):1557-1556, 2019.

SUMMARY: Plastination is an anatomical technique of cadaveric conservation created in 1977 by Gunther von Hagens, in Heidelberg, Germany, and that substitutes biological and / or fixation fluids with acetone, to then impregnate the samples with different resins, depending on the developed plastination technique, to finally carry out the polymerization of the components incorporated into the samples, to obtain dry and totally durable biological samples. The aim of this work was to develop a sheet plastination protocol with polyester resin (Biodur ${ }^{\circledR} \mathrm{P} 40$ ) in $3 \mathrm{~mm}$ thick slices of human brain. The samples were fixed and preserved with $10 \%$ formalin. The brains were sectioned with a slice cut machine, obtaining thin sheets of $3 \mathrm{~mm}$ thick. Immediately the slices of brain were placed in dehydration in $100 \%$ acetone, at $-25^{\circ} \mathrm{C}$, for 7 days the first acetone bath, and for another 3 more days, for the second acetone bath. Once the cuts were dehydrated, they were placed in Biodur ${ }^{\circledR} \mathrm{P} 40$ polyester resin and the forced impregnation was carried out in a vacuum chamber at room temperature $\left(20^{\circ} \mathrm{C}\right)$. Once the forced impregnation was finished, the curing stage was carried out, which first consists in the assembly of the curing chambers within which the slices with polyester resin were placed. The curing chambers were placed under UV light to accelerate the polymerization of the polyester and finished the plastination process. A sheet plastination protocol with polyester resin was successfully developed in the Laboratory of Plastination and Anatomical Techniques of Universidad de La Frontera, obtaining excellent conservation of brain slices, with differentiation of gray and white substances, and conservation of all morphological characteristics.

KEY WORDS: Sheet Plastination; Polyester resin. 


\section{REFERENCIAS BIBLIOGRÁFICAS}

Barnett, R. J. Plastination of Coronal and horizontal brain slices using the P40 technique. J. Int. Soc. Plastination, 12(1):33-6, 1997.

Gao, H.; Liu, J.; Yu, S. \& Sui, H. A new polyester technique for sheet plastination. J. Int. Soc. Plastination, 21:7-10, 2006.

Ottone, N. E. Gunther von Hagens, Creador de la plastinación. Reseña histórica y desarrollo de la técnica. Rev. Argent. Anat. Online, 4(2):706, 2013.

Ottone, N. E. Plastination: Techniques fundamentals and implementation at Universidad de La Frontera. J. Health Med. Sci., 4(4):293-302, 2018.

Ottone, N. E.; Baptista, C.; Latorre, R.; Bianchi, H. F.; del Sol, M. \& Fuentes, R. E12 sheet plastination - Techniques and applications. Clin. Anat., 31(5):742-56, 2018b

Ottone, N. E.; Cirigliano, V.; Bianchi, H. F.; Medan, C. D.; Algieri, R. D.; Borges Brum, G. \& Fuentes, R. New contributions to the development of a plastination technique at room temperature with silicone. Anat. Sci. Int., 90(2):126-35, 2015.

Ottone, N. E.; Cirigliano, V.; Lewicki, M.; Bianchi, H. F.; Aja Guardiola, S.; Algieri, R. D.; Cantin, M. \& Fuentes, R. Plastination Technique in laboratory rats: An alternative resource for teaching, surgical training and research development. Int. J. Morphol., 32(4):1430- 5, 2014.

Ottone, N. E.; del Sol, M. \& Fuentes, R. Report on a sheet plastination technique using commercial epoxy resin. Int. J. Morphol., 34(3):103943, 2016.

Ottone, N. E.; Vargas, C. A.; Veuthey, C.; del Sol, M. \& Fuentes, F. Epoxy sheet plastination on a rabbit head-new faster protocol with Biodur ${ }^{\circledR}$ E12/E1. Int. J. Morphol., 36(2):441-6, 2018a.

Prieto, R.; Vargas, C. A.; Veuthey, C.; Aja-Guardiola, C. \& Ottone, N. E. Fundamental concepts of the modified room temperature plastination protocol with silicone, with subsequent pigmentation, and its application for the conservation of human placenta. Int. J. Morphol., 37(1):375-6, 2019.

Reed, R. B.; Helms, J. \& Rowe, J. A. Curing times of P40 exposed to different light sources. J. Int. Soc. Plastination, 28:25-9, 2008.

Sora, M. C. \& Brugger, P. P40 brain slices plastination using methanol for dehydration. J. Int. Soc. Plastination, 15(1):22-4, 2000.

von Hagens, G.; Tiedemann, K. \& Kriz, W. The current potencial of plastination. Anat. Embryol. (Berl.), 175(4):411-21, 1987.
Dirección para correspondencia:

Prof. Dr. Nicolás Ernesto Ottone

Laboratorio de Plastinación y Técnicas Anatómcias

Centro de Investigación en Ciencias Odontológicas (CICO)

Facultad de Odontología

Universidad de La Frontera

Av. Francisco Salazar 01145

Casilla 54-D

Temuco

CHILE

\section{Email: nicolas.ottone@ufrontera.cl}

Recibido : 12-04-2019

Aceptado: 26-06-2019 